Beaulieu, Jean-Philippe, « Lettre de femme, voix d'homme ? Jeux identitaires et effets de travestissement dans la treizième épître familière d'Hélisenne de Crenne », Tangence, n 84, 2007, p. 31-47.

\title{
Lettre de femme, voix d'homme? Jeux identitaires et effets de travestissement dans la treizième épître familière d'Hélisenne de Crenne
}

\author{
Jean-Philippe Beaulieu, \\ Université de Montréal
}

\begin{abstract}
La dernière des treize lettres familières qui forment le premier volet des Epistres familieres et invectives (1539) reste à ce jour la missive la plus difficile à interpréter du recueil d'Hélisenne de Crenne. Son caractère cryptique, la voix masculine qu'y fait entendre l'épistolière et la présence d'allusions amoureuses assurent un caractère singulier à ce texte dont le fonctionnement peut se comprendre - c'est l'hypothèse que propose cet article - comme le résultat d'un travestissement identitaire (une femme s'y exprime à la manière d'un homme), doublé d'un travestissement générique (la lettre familière cache une lettre d'amour). L'expression directe du désir amoureux ne semble possible, dans le recueil, que par ce jeu de travestissements où la rhétorique masque et dévoile tout à la fois, en établissant entre épistolière et destinataire une familiarité affective dont l'existence ne peut être révélée au lecteur que sur le mode du simulacre.
\end{abstract}

On s'est récemment penché sur certains des phénomènes de travestissement qui, dans le premier ouvrage publié sous le nom d'Hélisenne de Crenne ${ }^{1}$, les Angoysses douloureuses qui procedent d'amours (1538), permettent à la voix auctoriale de se moduler au

1. Notons que l'attribution de ce texte à une figure féminine a récemment été mise en doute - sans pourtant être invalidée, à mon sens — par Anne RéachNgô, «La mise en livre des narrations de la Renaissance. Écriture éditoriale et herméneutique de l'imprimé», thèse de doctorat, Paris, Université de ParisSorbonne/Paris IV, 2005, p. 399-414.

Tangence, $\mathrm{n}^{\circ} 84$, été 2007, p. 31-47. 
féminin (dans la première partie du récit) et au masculin (dans les deuxième et troisième parties), de manière à décrire successivement l'expérience amoureuse d'une femme et celle d'un homme ${ }^{2}$. Une modulation analogue semble se retrouver dans les Epistres familieres et invectives (1539), lorsque, dans sa treizième lettre familière, Hélisenne, jusque-là l'unique scriptrice, prête momentanément sa plume à une voix masculine. La rubrique-argument qui précède la lettre indique en effet que l'épistolière y sert de truchement à un gentilhomme qui «aspiroit de rendre certain de ses nouvelles ung sien fidele compaignon ${ }^{3}$ ». Dans la mesure où le contenu de la missive «estoit digne d'estre en perpetuel silence conservé» (EFI, p. 97), la lettre fut rédigée de telle sorte qu'elle ne soit compréhensible que pour le destinataire. Ainsi soumise à un mécanisme de voilement tant de l'identité du locuteur que du sens du message, cette épître cryptique demeure à ce jour la portion la moins étudiée du recueil.

Pourtant, la fonction de la treizième épître s'éclaire lorsqu'on considère que ce texte cherche avant tout à attirer l'attention du lecteur sur la mise en scène épistolaire elle-même plutôt que sur le contenu du message, probablement voué à rester indéchiffrable, mais où se font entendre des inflexions amoureuses, inattendues dans le contexte d'une épître en prose dite familière. Il me semble que l'opacité qui caractérise cette lettre peut être interprétée comme un travestissement à la fois de l'identité du locuteur et du genre épistolaire utilisé ${ }^{4}$. D'une part, une femme y énonce un discours censément masculin; de l'autre, le registre familier cache une lettre dont on peut présumer, sans pouvoir le prouver, qu'elle

2. Cathleen Bauschatz, "Travestissement textuel dans la "Seconde Partie" des Angoysses douloureuses», dans Jean-Philippe Beaulieu et Diane DesrosiersBonin (sous la dir. de), Hélisenne de Crenne. L'écriture et ses doubles, Paris, Honoré Champion, 2004, p. 55-70.

3. Hélisenne de Crenne, Les Epistres familieres et invectives, édition de JeanPhilippe Beaulieu et Hannah Fournier, Montréal, Presses de l'Université de Montréal, 1995, p. 97. Dorénavant, les renvois à cet ouvrage seront indiqués par le sigle EFI, suivi de la page, et placés entre parenthèses dans le corps du texte.

4. Il s'agit bien évidemment d'une acception métaphorique du terme travestissement, qui renvoie ici à des phénomènes strictement textuels. À propos de l'histoire culturelle du travestissement, on consultera Sylvie Steinberg, La confusion des sexes. Le travestissement de la Renaissance à la Révolution, Paris, Fayard, 2001; de même que l'ouvrage collectif récemment paru sous la direction de Guyonne Leduc, Travestissement féminin et liberté(s), Paris, L'Harmattan, 2006. 
est de nature amoureuse. Dans les deux cas, le texte opère un brouillage des codes d'appartenance, conséquences de jeux de travestissement que la présente étude vise à éclairer en tant que manifestation problématique du dévoilement de soi. En effet, s'il s'agit bien d'une lettre d'amour déguisée, ce qui s'y exprime relève d'un registre qui ne trouve aucune autre place dans ce recueil, où il est question d'amour - pour le fustiger (épîtres familières 5 et 8), le confesser (épîtres familières 10 et 11) ou le nier (épîtres invectives 1 et 3) -, mais où, conformément aux catégories génériques annoncées par le titre, ne figure aucun discours amoureux. Dans une perspective d'intertextualité propre au corpus hélisénien ${ }^{5}$, le recours au subterfuge du travestissement en tant que véhicule de l'expression codée du sentiment amoureux doit probablement se comprendre comme le prolongement des problèmes de communication (dissimulations, détractions, faux rapports, malentendus) qui, dans les Angoysses, marquent la relation illicite - et censément secrète - entre Hélisenne et Guenelic. Tout en invitant à une telle lecture contextuelle, qui permet à l'imagination de combler les vides référentiels du texte, le travestissement refuse néanmoins le plein dévoilement de son sens, comme si la lettre amoureuse ne pouvait être rendue publique qu'en conférant au lecteur le statut de voyeur. Éminemment conventionnelle aux XVI ${ }^{e}$ et $\mathrm{XVII}^{\mathrm{e}}$ siècles $^{6}$, la lettre d'amour trouve ici une interprétation ludique où le travestissement voile le sens de l'épître tout en mettant en scène l'ambiguité d'une rhétorique amoureuse qui se déclare par le simulacre d’un lien épistolaire «familier».

\section{Lectures et relectures}

Ce voilement du sens a été signalé par les commentaires critiques portant sur cette lettre, fort peu nombreux et substantiels en comparaison avec les études consacrées aux épîtres invectives ${ }^{7}$. Constatant le caractère cryptique de la treizième lettre familière,

5. Jean-Philippe Beaulieu et Diane Desrosiers-Bonin, «Les jeux du même, de l'autre et du multiple chez Hélisenne de Crenne», dans Hélisenne de Crenne, ouvr. cité, p. 12-14.

6. Jacqueline Boucher, "L'amour à la cour de Henri III et Henri IV : le discours et le réel », dans Les visages de l'amour au XVII siècle, Toulouse, Université de Toulouse-Le Mirail, 1984, p. 18.

7. On se reportera aux travaux de Jerry C. Nash, notamment «Renaissance Misogyny, Biblical Feminism, and Helisenne de Crenne's Epistres familieres et invectives ", Renaissance Quarterly, New York, vol. L, n 2, 1997, p. 379-410. 
sans trop s'attarder à ses aspects problématiques, ces commentaires s'appuient sur le préambule descriptif que propose la rubriquerésumé et que je reproduis ici intégralement:

Epistre par ma dame Helisenne composée, laquelle elle fist à l'instante priere d'ung gentil homme, qui tres affectueusement aspiroit de rendre certain de ses nouvelles ung sien fidele compaignon: mais pource que la chose dont, il le vouloit advertir, estoit digne d'estre en perpetuel silence conservée, Il requist que escripte fut si occultement, qu'a nulz excepté son compaignon elle fut intelligible (EFI, p. 97).

Ainsi, dans leur traduction anglaise du recueil, Mustacchi et Archambault laissent entendre que la lettre aurait été écrite par Hélisenne à la demande d'un homme voulant qu'elle envoie des renseignements sur elle-même et sa passion amoureuse à un ami non identifié. Cette lecture repose sur une interprétation intéressante du possessif dans l'expression «ses nouvelles», qu'il faudrait ainsi comprendre comme se rapportant non au gentilhomme, mais à Hélisenne elle-même ${ }^{8}$. À partir de cette prémisse, les traducteurs affirment, sans en faire la démonstration, qu'Hélisenne aurait rédigé une lettre codée à l'intention de son amant ${ }^{9}$. Dans l'édition du recueil qu'il a fait paraître en 1996, Jerry Nash reprend la même idée en affirmant que cette lettre correspond à "la cordialle et fidele amour" d'Hélisenne envoyée à son amant par l'intermédiaire d'un de ses compagnons", sans trop s'attarder au fonctionnement d'une telle médiation épistolaire ${ }^{10}$. Dans le même sens, Luc Vaillancourt privilégie une interprétation amoureuse de cette missive: "La dernière épître "familière", composée par Hélisenne au masculin, semble être un message codé destiné à son amant. Elle lui révèle toutes les souffrances endurées pour lui et l'assure de la constance de son amour ${ }^{11}$.» Les motifs d'une telle lecture restent cependant imprécis. Il est vrai que les possibilités interprétatives que nous offre cette épître sont limitées. Si la lettre est «escripte [...] si occultement» pour n'être compréhensible que pour le destinataire, à l'exclusion de tout autre lecteur, comment

8. A Renaissance Woman. Helisenne's Personal and Invective Letters, Marianna M. Mustacchi et Paul J. Archambault (trad.), Syracuse (New York), University of Syracuse Press, 1986, p. 69.

9. A Renaissance Woman, ouvr. cité, p. 6.

10. Hélisenne de Crenne, Les Epistres familieres et invectives, édition de Jerry C. Nash, Paris, Honoré Champion, 1996, p. 29.

11. Luc Vaillancourt, La lettre familière au XVI siècle. Rhétorique humaniste de l'épistolaire, Paris, Honoré Champion, 2003, p. 195. 
peut-on espérer la décoder? Comment appréhender une rhétorique épistolaire qui met à mal la triangularité entre l'épistolier, le destinataire et le lecteur que suppose tout recueil destiné à la publication ${ }^{12}$ ? Si, dans des études antérieures, je me suis surtout arrêté au mécanisme de refus de sens qu'exhibe la lettre ${ }^{13}$, le temps est venu de forcer ce mécanisme en documentant l'hypothèse d'une lettre amoureuse travestie.

\section{Une singulière épître familière}

Tout juste avant les cinq épîtres invectives formant le deuxième volet du recueil, la treizième et dernière lettre familière se présente comme un hapax au cœur même d'un ensemble de textes épistolaires dont la finalité est généralement claire, qu'il s'agisse de consolatio ou d'exhortatio dans les premières lettres familières, ou d'objurgatio dans les invectives ${ }^{14}$. Parmi les aspects de cette lettre qui concourent à son indétermination sémantique, constatons tout d'abord l'identité apparemment masculine du locuteur, si l'on se fie aux marques de "généricité" (au sens de gender) qui lui sont associées. Ainsi en est-il de l'usage systématique du masculin dans l'accord des participes passés et des adjectifs. Par exemple, lorsque l'épistolier affirme au début de la lettre: «ceste veritable opinion [...] te faict estimer que je soye plus stimulé, et violentement pressé » (EFI, p. 97), les participes "stimulé » et "pressé » sont accordés au masculin. Dans la mesure où l'auteure n'hésite pas, ailleurs, à utiliser le féminin dans des contextes similaires, il est clair que l'on veut donner à entendre que l'épistolier est un homme, tout comme le destinataire, désigné par l'expression "mon amy». Loin d'être accidentel, l'usage du masculin est confirmé par les autres références de la lettre où le locuteur dit

12. Au sujet de l'inscription du lecteur dans le processus de publication des recueils épistolaires, voir Janet Gurkin Altman, «The Letter as a Literary Institution 1539-1789: Toward a Cultural History of Published Correspondences in France», Yale French Studies, New Haven (Connecticut), n ${ }^{\circ} 71$, 1986, p. 18-19.

13. Jean-Philippe Beaulieu, «La dispositio du recueil épistolaire: unité et variété dans les Epistres d'Hélisenne de Crenne», dans Marie-Claude Malenfant et Sabrina Vervacke (sous la dir. de), "Écrire et conter». Mélanges de rhétorique et d'histoire littéraire du XVI siècle offerts à Jean-Claude Moisan, Québec, Presses de l'Université Laval, 2003, p. 47-48.

14. Jean-Philippe Beaulieu, «La dispositio du recueil épistolaire», art. cité, p. 4445. 
appartenir au sexe masculin, notamment lorsqu'il soupçonne son destinataire de «mentalement [l'] accuser d'estre homme de petite consideration» (EFI, p. 97).

Curieusement, le lien entre l'épistolier et son destinataire est tributaire d'images davantage associées à une relation amoureuse qu'à un lien d'amitié, au sens moderne du terme. Se disant «aveuglé d'affection», l'épistolier parle de la «cordialle et fidele amour» qu'il porte au destinataire (EFI, p. 97); plus loin, il affirme avoir aimé celui-ci d'une "amour fervente» (EFI, p. 98). Si certaines expressions peuvent évidemment s'appliquer à l'amitié ${ }^{15}$, elles sont associées à un sentiment d'excès qui, dans les lettres précédentes, affectent la description du sentiment amoureux mais pas la relation avec le destinataire, qui est de nature amicale comme nous le verrons plus loin. Ainsi, lorsque l'épistolier affirme: «O [...] combien excessive est l'inveterée amytié, en mon cueur inserée» (EFI, p. 98), il semble parler d'autre chose que d'amitié, ce que confirme, à la page suivante, la référence à «l'invincible puissance d'amour» en rapport avec la détermination imprudente de son correspondant en ce qui a trait à un projet les concernant tous deux et comportant des risques pour le destinataire:

Tu dis estre appareillé d'empenner ton obeissance pour accomplir cela que tu estimerois m'estre aggreable et acceptable, en peine de succumber au mesme peril du temeraire Icarus. Je te certifie que rememorant tes discrettes parolles par toy à moy exprimées, tu me donne merveille. Mais congnoissant l'invincible puissance d'amour, je te croy: toutesfois si la verité tu veulx dire premier que venir à ceste determination, tu as senti ung merveilleux debat en toy entre ton altissime prudence et ton excessif amour (EFI, p. 99).

À moins d'être interprétées comme l'expression - peu probable — du désir homosexuel, de telles références allusives liant deux hommes sont d'autant plus curieuses que l'épistolier, au risque d'un certain efféminement ${ }^{16}$, se décrit un peu plus tôt au

15. Dans le contexte de l'époque, on peut entendre "amour» dans un sens qui désigne un élan affectif ayant des objets très variés (voir Maurice Daumas, $\mathrm{La}$ tendresse amoureuse XVIe-XVIII siècles, Paris, Perrin, 1996, p. 82). Ainsi, dans le Tristan de Pierre Sala (rédigé vers 1525-1527), l'amitié naissante entre Lancelot et Tristan est décrite comme une "si tres grande rage d'amour", dans le cadre d'une scène dont la finalité, cependant, est peut-être comique (Tristan, éd. de Lynette Muir, Genève, Droz, 1956, p. 35).

16. Il s'agit d'un processus différent de l'«effémination» de l'épistolier masculin qu'Alain Roger constate dans des corpus plus tardifs et résultant d'un désir de 
moyen d'une métaphore amoureuse généralement associée à la femme: «[...] car tu scais indubitablement que j'aspire trop plus à te livrer le chasteau, par toy assiegé, que toy mesmes n'as de desir de le posseder» (EFI, p. 97). Bien que la notion d'amitié renvoie à l'époque à une charge émotive qui peut être confondue avec l'amour ${ }^{17}$, l'usage de ce type d'image suggère un cadre relationnel amoureux, hypothèse que renforcent les formules de rapport au destinataire qui, dès l'incipit de la lettre, lient de façon serrée le «je» et le « tu ${ }^{18} »$, établissant un degré de proximité plus grand que dans les autres lettres familières:

Après avoir quelque temps consumé en la consideration de tes escriptz, je puis facilement conjecturer, que tu te persuade que je te veuille increper, te attribuant le vice de pusillanimité, Toutefois je te certifie que jamais telle chose en ma pensée ne tint lieu d'occupation (EFI, p. 97 ; je souligne).

Que cette intimité soit sujette au secret n'est guère surprenant quand on pense que, dans la neuvième épître, Hélisenne s'adresse à son amie Clarice en l'incitant à «ne faire indice de [son] amoureuse flamme» (EFI, p. 84), soulignant du même coup la vulnérabilité de la passion féminine face à l'opinion publique. Sous cet éclairage, il est compréhensible que la force de la "fervente amour» (EFI, p. 98) qu'évoque la treizième épître implique qu'elle doive être à la fois confiée et dissimulée, dispositions contradictoires qui nous renvoient à ce qui est au cœur même de la dynamique amoureuse du personnage d'Hélisenne dans la première partie du roman de $1538{ }^{19}$.

se rapprocher de sa "proie» pour mieux la séduire (Alain Roger, «La lettre d'amour et l'effémination épistolaire», dans Jean-Louis Bonnat et Mireille Bossis (sous la dir. de), Écrire, publier, lire les correspondances, Nantes, Publications de l'Université de Nantes, 1980, p. 93-104).

17. Maurice Daumas, La tendresse amoureuse, ouvr. cité, p. 101.

18. Un peu à la manière du discours que la religieuse adresse à son amant dans les Lettres portugaises de Guilleragues (Susan Lee Carrell, Le soliloque de la passion féminine ou le dialogue illusoire, Tübingen, Gunter Narr Verlag, 1982, p. 12).

19. Martine Debaisieux, "Subtilitez féminines". L'art de la contradiction dans l'œuvre d'Hélisenne de Crenne», Études littéraires, Québec, vol. 27, n 2, 1994, p. 27-30. 


\section{Du côté des Angoysses}

C'est d'ailleurs probablement en effectuant une lecture croisée des Angoysses et des Epistres que peut s'éclairer davantage le caractère énigmatique de la lettre qui nous intéresse. Cette lecture est facilitée et encouragée par le fait que les Epistres, qui n'ont connu qu'une édition en texte indépendant (1539), ont été intégrées à partir de 1543 dans le recueil des Euvres d'Hélisenne, favorisant, dès lors, une lecture conjointe et continue de ce corpus. Dans un premier temps, en raison de la présence de Quezinstra comme destinataire de la douzième lettre, on est tenté de reconnaître, dans ces figures masculines laissées sans identité précise ${ }^{20}$, les compagnons d'aventure que sont Quezinstra et Guenelic dans les Angoysses. Toutefois, la rubrique-argument et la nature même du discours tenu par le scripteur nous invitent à considérer que cette relation amicale en cache une autre de nature amoureuse, d'autant que, dans certaines des lettres familières qui précèdent, le sentiment amoureux féminin est évoqué ou avoué. Ainsi, les lettres 5, 8 et 9 font état de l'amour de Galazie et de Clarice. Quant aux lettres 10 et 11, elles se veulent une confession adressée à Galazie de la "cupidité venericque»(EFI, p. 86) à laquelle Hélisenne dit désormais être soumise et qui, pour tout lecteur du corpus hélisénien, convoque forcément la figure de Guenelic, même si ce personnage n'est jamais nommé dans le recueil. À la lumière de cet implicite contextuel, il semble possible de considérer que de la même façon qu'Hélisenne, dans les deuxième et troisième parties du roman, "parle en la personne de son amy Guenelic ${ }^{21}$ », assurant le relais qui permet à celui-ci de devenir le narrateur, l'épistolière fait appel, dans la treizième épître, à une forme de ventriloquie par laquelle elle contrefait la voix d'un homme pour adresser un discours amoureux à un amant dans lequel il est loisible de reconnaître Guenelic. C'est ce que nous engagent à croire, dans la lettre, les divers rappels de la relation amoureuse des Angoysses: la peur de la divulgation, l'incompréhension mutuelle, les supputations quant à la conduite de l'autre, le rôle de la jalousie, et même la séquestration d'Hélisenne par son mari, évoquée dans la douzième

20. Contrairement aux autres lettres familières, où les destinataires sont désignés nommément ou par leur lien — souvent de parenté — avec l'épistolière.

21. Hélisenne de Crenne, Les angoysses douloureuses qui procedent d'amours, édition de Christine de Buzon, Paris, Honoré Champion, 1997, p. 227. Les renvois ultérieurs à cet ouvrage seront indiqués par le sigle $A N$, suivi de la page, et placés entre parenthèses dans le corps du texte. 
lettre, où l'épistolière exhorte Quezinstra à ne pas «discontinuer les remonstrances» faites à son époux dans le but de convaincre celui-ci que c'est à tort qu'il lui "preste matiere de [1]'angustier et adolorer» (EFI, p. 96).

Par sa forme même et son contenu allusif, la treizième épître familière semble transposer la communication problématique qui hante la première partie du roman. On sait que, dans cette partie des Angoysses, les relations entre les amants sont soumises aux aléas d'échanges difficiles, marqués par la dissimulation ou le mensonge, et fortement influencés par les commérages, détractions et faux rapports ${ }^{22}$. À l'instar de la difficulté qu'éprouve Hélisenne, dans le roman, à interpréter la conduite de son amant, le scripteur de la dernière épître familière semble peiner à comprendre le comportement du destinataire. Dès le début de la lettre, cela se traduit par la présence de nombreuses formules prudentes, par lesquelles l'épistolier suggère des hypothèses, des opinions dont la valeur est relative. Le doute plane sur ce que l'on pense de l'autre et sur ce qu'on pense que l'autre pense de soi, d'où l'usage de verbes comme conjecturer, attribuer, impropérer, souvent associés à des conditionnels, comme l'illustre bien l'incipit de la lettre qui est reproduit plus haut (EFI, p. 97).

C'est à la lumière de ce doute qu'il faut comprendre le reproche de pusillanimité, adressé au destinataire, qui, dès le début de la lettre, n'est pas sans évoquer la tiédeur amoureuse qu'Hélisenne reproche à Guenelic dans le roman ${ }^{23}$. Bien que, dans la treizième épître, le scripteur formule ce reproche en affirmant n'avoir jamais pensé à une telle chose («[...] je te certifie, que jamais telle chose [le vice de pusillanimité] en ma pensée ne me tint occupation» [EFI, p. 97]), la seule présomption de la faute suffit à entacher le destinataire. Comme Guenelic dans le roman, ce dernier semble partagé entre son désir et l'inquiétude qu'il éprouve quant aux conséquences de celui-ci; on dit à son propos qu'il sent en lui

22. Au sujet de la dissimulation dans les Epistres et les Angoysses, voir Diane S. Wood, Helisenne de Crenne. At the Crossroads of Renaissance Humanism and Feminism, Madison (Wisconsin) et Teaneck (New Jersey)/Londres, Fairleigh Dickinson University Press/Associated University Presses, 2000, p. 89-92.

23. Au début de la deuxième partie des Angoysses, Hélisenne va même se sentir obligée d'expliquer pourquoi Guenelic, amant peu méritoire, a par la suite «enduré tant de fatigues pour sa Dame retrouver» ( $A N$, p. 230), faisant ainsi preuve d'un courage supérieur à sa «basse condition» $(A N$, p. 229). 
«ung merveilleux debat» entre son "altissime prudence» et son «excessif amour» (EFI, p. 99). Par contraste, soupçonné par son destinataire d'être «aveuglé d'affection » et «violentement pressé par temeraire hardiesse» (EFI, p. 97), l'épistolier nous fait penser au personnage d'Hélisenne dans le roman, rendue excessive par l'amour qui la fait agir "comme femme du tout alienée de raison" (AN, p. 118). Lourd d'«inconveniens futurs» (EFI, p. 98), le projet commun des correspondants s'exprime à travers la métaphore déjà évoquée du château assiégé par le destinataire, l'épistolier se disant emprisonné ( «si j'estoye en ma liberté»; EFI, p. 102) et sous la surveillance d'un "vigilant et soliciteux gardien» (EFI, p. 98). Cette situation évoque évidemment la détention d'Hélisenne dans le château de Cabasus, d'autant que le gardien en question, un «cler voyant Argus", est accompagné d'une «infelice et mauldicte creature» (EFI, p. 102), dont la description n'est pas sans rappeler la dame maldisante qui s'applique à "molester et aggraver [1] es tourmens » d'Hélisenne à Cabasus ( $A N$, p. 425). De plus, la difficulté évoquée à la fin de la lettre quant à sa transmission, en raison «de la continuele assistance de la predicte abhominable creature» (EFI, p. 103), c'est-à-dire la dame maldisante, renvoie aux obstacles qui, dans la troisième partie du roman, retardent la rencontre des amants et les contraignent à user du «benefice litteraire» - le commerce épistolaire - pour communiquer l'un avec l'autre (AN, p. 433).

Ces indices, de nature intertextuelle, nous suggèrent donc une analogie avec la situation amoureuse du roman, analogie préparée par les lettres précédentes et qui nous dispose à voir Hélisenne non pas seulement comme un simple "prête-plume», mais comme la scriptrice véritable de cette lettre, cherchant à dissimuler la nature de ses sentiments, comme elle tente de le faire si souvent dans le roman $^{24}$.

\section{Un discours amoureux travesti}

Admettre l'hypothèse d'un tel travestissement identitaire implique de considérer que cette lettre censément familière connaît un infléchissement vers la lettre amoureuse, mais selon une modalité qu'il est difficile de cerner. Si la lettre familière a fait l'objet de nets efforts de théorisation dans divers traités épistolographiques de la

24. Face à son mari, à son entourage et même à son amant, à qui elle décide de ne pas «declairer le secret de [s] on cueur» pour «plus ardentement l'enflamber» (AN, p. 167). 
première moitié du $\mathrm{XVI}^{\mathrm{e}}$ siècle ${ }^{25}$, la seconde, en dépit de ce à quoi on s'attendrait, est plus problématique d'un point de vue définitoire ${ }^{26}$. Selon les manuels d'art épistolaire et les secrétaires qui se développent à la Renaissance ${ }^{27}$, la lettre d'amour paraît plus strictement codifiée que la lettre familière, puisque celle-ci est vouée, selon les prescriptions érasmiennes, à s'adapter à de multiples situations et correspondants ${ }^{28}$. Ce codage, qui emprunte une bonne partie de ses conventions à la tradition de la lyrique pétrarquiste ${ }^{29}$, s'affiche, dans les recueils de lettres amoureuses antérieurs ou contemporains à l'ouvrage d'Hélisenne, par l'usage de l'épître en vers qui rend ostensible la littérarité de la lettre amoureuse ${ }^{30}$.

Dans les Angoysses, Hélisenne privilégie pour sa part l'épître amoureuse en prose, en s'inspirant directement des lettres qui figurent dans la traduction française du Peregrin de Jacopo Caviceo $^{31}$. Ces lettres que s'adressent les amants optent pour une rhétorique amoureuse manifeste dont le contexte narratif vient en

25. À ce sujet, voir Luc Vaillancourt, La lettre familière, ouvr. cité, p. 147-165; et Guy Gueudet, L'art de la lettre humaniste, textes réunis par Francine Wild, Paris, Honoré Champion, 2004, p. 247-273.

26. Luc Vaillancourt affirme à ce sujet que le «statut de la lettre amoureuse à la Renaissance est loin d'être évident » (La lettre familière, ouvr. cité, p. 25).

27. Surtout à partir de la deuxième moitié du XVI e siècle (Maurice Daumas, La tendresse amoureuse, ouvr. cité, p. 108-109).

28. Jacques Chomarat, Grammaire et rhétorique chez Érasme, Paris, Les Belles Lettres, 1981, vol. II, p. 1010-1012.

29. Sur le caractère "artificieux" (parce qu'italianisant) de la lettre d'amour à la Renaissance, voir Bernard Bray, L'art de la lettre amoureuse: des manuels aux romans (1550-1700), La Haye, Mouton, 1967, p. 10. Yves Giraud décrit les effets convenus de certains ouvrages comme les Lettres amoureuses (1602) d'Antoine Du Périer ( La dimension épistolaire dans quelques ensembles épistolaires du XVI ${ }^{\mathrm{e}}$ siècle", dans Michèle Clément et Pascale Mounier (sous la dir. de), Le roman français au $X V I^{\text {e }}$ siècle ou le renouveau d'un genre dans le contexte européen, Strasbourg, Presses universitaires de Strasbourg, 2005, p. 85).

30. Pensons par exemple aux Epistres veneriennes de Michel d'Amboise, Paris, Jehan Longis et Denis Janot, 1534. Soulignant la façon dont l'épître amoureuse en vers est tributaire des conventions de la poésie lyrique, Thomas Sébillet, au chapitre VII de son Art poétique (1548), assimile ce type d'épître à l'élégie. Pour un survol de la pratique de l'épître en vers en France, voir Marc Bizier, Les lettres romaines de Du Bellay. Les Regrets et la tradition épistolaire, Montréal, Presses de l'Université de Montréal, 2001, p. 53-59.

31. Diane S. Wood, «Les lettres d'Hélisenne de Crenne et Jacopo Caviceo : lecture et stratégies scripturaires", dans Hélisenne de Crenne, ouvr. cité, p. 131-152. Notons que, dès le $\mathrm{Xv}^{\mathrm{e}}$ siècle, divers ouvrages narratifs espagnols et italiens font appel à la lettre d'amour pour alimenter le récit. À ce sujet, voir Laurent Versini, Le roman épistolaire, Paris, Presses universitaires de France, 1979, p. 14-17. 
outre préciser l'orientation ${ }^{32}$. Le parti pris de la lettre 13 est tout autre: l'idée de code doit y être comprise au sens de chiffre, de cryptage. L'imprécision du cadre référentiel rend difficile l'interprétation de la rhétorique qui cherche à s'y exprimer ${ }^{33}$. N'est-ce pas parce qu'il y est question de cette "amour vitieuse», dont parle Pierre Fabri dans la section sur les «lettres missives» de son traité, répugnant à donner des exemples d'expression épistolaire de ce sentiment, comme s'il valait mieux le passer sous silence ${ }^{34}$ ? $\mathrm{Si}$, sous l'Ancien Régime, ainsi que l'affirme Maurice Daumas, "[é]changer des lettres est au fond une façon de faire l'amour à distance, donc de conserver une certaine honnêteté", il est assez normal que la lettre d'amour opte ou bien pour l'étalage du sentiment amoureux ou bien pour le secret et la prudence ${ }^{35}$, en pratiquant un langage hermétique ou «hiéroglyphique ${ }^{36} »$. Ce désir de secret dans les échanges amoureux est clairement exprimé par Alessandro Piccolomini dans la section ayant pour manchette "Que deux personnes amantes doivent parler en tierce personne de leurs affections et desirs ${ }^{37}$ », de son Dialogo della creanza de le donne (1539), selon la formulation de la version française procurée par François d'Amboise:

Souventesfois en compagnie [les personnes amantes] pourront descouvrir la condition de leur pensée, et l'estat de leur amitié l'un à l'autre par noms feints et empruntez, qui se trouvent en Amadis et aux Romans : et faisant semblant de conter des nouvelles et fables, tenir un langage qui ne s'entend que d'eux, lequel ne sera prins des autres qui l'escouteront que pour contes de Chevaliers errans ou fables ${ }^{38}$.

32. Notons toutefois que le contenu de ces lettres n'est pas au-dessus de tout soupçon. En effet, le personnage d'Hélisenne fait preuve de circonspection quant aux motivations réelles d'une des lettres de Guenelic en se demandant si celle-ci n’a pas été «par simulation ou fainctise composée» (AN, p. 132).

33. Mais dont on peut sentir la nature partiellement exhortative ou «suasoire». Concernant les genres épistolaires selon la typologie d’Érasme, on se reportera à Claude La Charité, La rhétorique épistolaire de Rabelais, Québec, Éditions Nota bene, 2003, p. 245-247.

34. Pierre Fabri, Le grant et vray art de pleine rhetoricque, réimpression de l'édition de 1521, Genève, Slatkine, 1972, fo 79, $\mathrm{v}^{\circ}$.

35. Maurice Daumas, La tendresse amoureuse, ouvr. cité, p. 116.

36. Voir l'emploi de l'expression «lettre hierogliphicque» que fait Gilles Corrozet dans l'adresse «Aux bons espritz et amateurs des lettres» qui figure au début de l'Hécatomgraphie, Paris, Denis Janot, 1540, fo Aiii, $\mathrm{v}^{\circ}$.

37. Alessandro Piccolomini, Dialogues et devis des damoiselles, pour les rendre vertueuses et bien-heureuses en la vraye et parfaicte amitié, François d'Amboise (trad.), Paris, V. Norment, 1581, fo 182 , $\mathrm{v}^{\mathrm{o}}$.

38. Alessandro Piccolomini, Dialogues et devis des damoiselles, ouvr. cité, fo $182, \mathrm{v}^{\circ}$. 
Il n'est guère difficile d'imaginer les conséquences, dans le champ épistolaire, d'un tel usage de la fiction et de la «feintise» dans le but de favoriser une communication cryptée entre les amants qui peuvent ainsi «faire entendre la qualité de leur passion» comme s'ils « avoient la commodité de parler seuls privément ensemble ${ }^{39}$ ». Doit-on se surprendre que, à la même époque, Michel d'Amboise ait intitulé l'un de ses ouvrages Le secret d'amours, mettant la pratique épistolaire amoureuse sous le signe du secret, même si, dans les faits, les vingt épîtres en prose qui figurent dans son livre ne sont en rien hermétiques ${ }^{40}$ ? Manifestement, l'important est ici de suggérer le secret plutôt que de l'actualiser dans les lettres.

À cet égard, dans le cadre d'un recueil épistolaire, la treizième lettre familière semble être l'un des rares exemples à son époque d'un réel chiffrage amoureux, qui illustre particulièrement bien l'expression "parler en tierce personne» qu'utilise Piccolomini, grâce à un discours indirect et controuvé qui est censé révéler à la personne concernée ce qu'il faut dissimuler à autrui ${ }^{41}$. La nécessité à la fois de taire et de révéler l'amour illicite est un motif récurrent dans le recueil, tout comme dans le roman, d'ailleurs, où l'écriture des lettres s'effectue dans le secret et avec la plus grande prudence (mais en vain puisque le mari finit par les découvrir) ${ }^{42}$. Dans la treizième lettre, la prudence semble extrême, mais on se demande bien qui elle vise. Qui veut-on écarter ou tromper: le lecteur du recueil ? Pourtant, depuis les lettres 10 et 11 , celui-ci est déjà au

39. Dans la dédicace à Catherine de Clermont qui ouvre ses Lettres amoureuses (Lyon, Paul Frellon et Abraham Cloquemin, 1595, p. 5), Étienne Du Tronchet évoque, entre autres considérations, la question de ce qui mérite d'être secrètement traité dans le cadre de la lettre amoureuse, puisque celle-ci permet de «declarer ouvertement ses privees intentions».

40. Le secret d'amours composé par Michel d'Amboise, ou sont contenues plusieurs lettres tant en rithme qu'en prose, fort recreatives à tous amans, Paris, Arnoul et Charles Langelier, 1542.

41. Certains romans de l'époque mettent en scène des lettres factices dont les effets de ventriloquie se rapprochent quelque peu de ce qui nous intéresse chez Hélisenne, mais dans un contexte qui n'est pas nécessairement amoureux. C'est le cas de la lettre d'Angiolo à l'abbesse que contrefait Agathe dans le Dialogue treselegant intitule le Peregrin, François d'Assy (trad.), Paris, [J. André], 1535, tiers livre, chap. 45-48, fo $269, \mathrm{v}^{\circ}-$ fo 272 , r. Le contexte narratif permet toutefois le plein dévoilement du stratagème en présentant successivement l'original et la fausse lettre.

42. C'est ce que l'on trouve aussi dans l'une des sources des Angoysses, La deplourable fin de Flamete, Maurice Scève (trad.), Paris, Denis Janot, 1536, où la rédaction des lettres amoureuses doit se faire dans le secret. 
courant du sentiment qu'éprouve Hélisenne. Ce n'est donc pas la confession amoureuse qui parait problématique, mais plutôt le fait d'exhiber un discours épistolaire proprement amoureux. Dans un ouvrage où, contrairement au roman, l'objet du désir n'est jamais nommé ou ouvertement convoqué, l'interprétation de la treizième épître comme lettre d'amour non assumée - puisqu'elle ne renonce pas à la désignation de lettre familière - attire notre attention moins sur son contenu que sur le décodage qu'on impose au lecteur. Le travestissement doit alors être compris non comme un déguisement parfait, qui occulte l'identité première, mais comme un masque partiel qui jette le doute sur la nature même de la lettre, en indiquant que le fait d'afficher le simulacre est probablement plus important que son plein dévoilement. De manière détournée, le registre familier s'investit alors d'un surplus de sens - absent du reste du recueil —, auquel contribue l'usage de l'érudition la plus marquée du groupe des lettres familières, ajoutant une couche supplémentaire de références à un ensemble déjà complexe et équivoque ${ }^{43}$. Si, dans les lettres invectives ou dans le Songe de 1540, l'érudition témoigne d'une volonté d'étaler un certain savoir et, du fait même, une ambition vraisemblablement humaniste ${ }^{44}$, les références analogiques à des figures antiques (Argus, Icare, Achille et Patrocle, Priam, César) ne semblent pas mises ici au service de l'efficacité rhétorique du discours; elles engagent plutôt ce dernier dans des avenues secondaires qui soulignent le caractère artificieux du discours. Notons le rappel de la thématique du secret et du cryptage que l'on peut sentir dans l'utilisation de la figure de Dédale, dont "l'artificielle ingeniosité» (EFI, p. 99), le labyrinthe, évoque l'effort à fournir pour déchiffrer le sens du texte et atteindre la sortie. Mais, comme le précise la fin de la lettre à propos des menaces qui pèsent sur l'épistolier et qui lui imposent un langage codé, «tout secret parler, est prevision de suspection» (EFI, p. 103). Par conséquent, le seul fait de pratiquer le cryptage révèle la présence d'un secret, à défaut d'en préciser la nature. Que nous reste-t-il, au moment de prendre congé, sinon cette «suspection», puisqu'on refuse au lecteur le partage de la familiarité des lettres précédentes, au profit d'un type d'intimité dont l'intelligibilité est problématique?

43. Jean-Philippe Beaulieu et Diane Desrosiers-Bonin, "Allégorie et épistolarité. Les jetées de l'érudition féminine chez Hélisenne de Crenne», Revue d'histoire littéraire de la France, Paris, vol. XCIX, nº 6, 1999, p. 1158-1159.

44. Robert D. Cottrell, «Le Songe d'Hélisenne de Crenne», dans Hélisenne de Crenne, ouvr. cité, p. 238. 


\section{Une rhétorique du simulacre?}

Si elle est en grande partie étrangère à la dialectique habituelle des recueils de lettres amoureuses, qui impose au sentiment amoureux une expression conventionnelle ${ }^{45}$, la rhétorique cryptée de la treizième épître familière a pourtant un effet similaire : masquer le caractère singulier de l'expression amoureuse par l'usage d'une rhétorique qui attire l'attention du lecteur sur la littérarité et les effets du texte. Dans le contexte contre-exemplaire du roman ${ }^{46}$, une telle volonté de dissimulation pourrait être interprétée comme la manifestation des débordements causés par l'amour, montrant jusqu'où va le désir de communiquer avec l'aimé ${ }^{47}$. Mais, dans le recueil, l'absence de cadre didactique voue essentiellement la treizième lettre à la seule monstration de la clandestinité. C'est entre les lignes qu'il faut lire l'essentiel: un aveu d'amour qui, au contraire de la confession amicale des lettres 10 et 11, n'arrive pas à se dire ouvertement. Réactivant le thème de la difficulté des amants à se parler, la lettre cryptée et les épîtres qui la précèdent inversent les données du roman, où la lettre amoureuse est possible mais où la confession à des tiers reste problématique. Ainsi, dans les Epistres, parler de cet amour à Galazie n'impose aucun codage, tandis que la confirmation de ce même amour dans une missive adressée à l'amant ne paraît guère envisageable, ne laissant à ce registre qu'un espace d'expression étroit et paradoxal qui n'en constitue pas moins le point culminant (mais «ténébreux», pour reprendre l'expression de Virginia Krause ${ }^{48}$ ) de la partie familière du recueil, faisant basculer celui-ci dans un ludisme qui cherche à rompre la stricte association entre femme et amour que les épîtres précédentes ont contribué à créer. Il n'est

45. Bernard Bray, L'art de la lettre amoureuse, ouvr. cité, p. 8-9.

46. Au sujet du dispositif de l'exemplarité dans le roman, voir Marie-Claude Malenfant, Argumentaires de l'une et l'autre espèces de femme. Le statut de l'exemplum dans les discours littéraires de la première moitié du XVI siècle, Québec, Presses de l'Université Laval, 2003, p. 424-426.

47. À propos de la nature pathologique de l'amour et de ses conséquences contradictoires, voir Michel Stanesco, «Mélancolie amoureuse et chevalerie dans Les Angoysses douloureuses d'Helisenne de Crenne", dans Emmanuel Bury et Francine Mora (sous la dir. de), Du roman courtois au roman baroque, Paris, Les Belles Lettres, 2004, p. 166-168.

48. Virginia Krause nous rappelle le caractère ombragé, obscur du secret amoureux qui, dans le roman, confine généralement son expression à des lieux privés ou clandestins ( ¿Confessions d’une héroïne: les Angoysses douloureuses d’Hélisenne de Crenne», dans Hélisenne de Crenne, ouvr. cité, p. 31-32). 
pas indifférent, à cet égard, que les épîtres invectives qui suivent maintiennent cette dissociation: Hélisenne y nie un sentiment amoureux que le lecteur sait pourtant être vrai, grâce à un déplacement du discours épistolaire vers des enjeux plus généraux qui touchent la valeur de la femme et son droit à l'écriture ${ }^{49}$. La rhétorique délibérative ${ }^{50}$ qui s'épanouit dans ce groupe de lettres est par conséquent fondée sur une morale douteuse, pratique qu'annonce à sa manière la treizième épître familière qui, en tant que point d'articulation des deux volets de l'ouvrage, nous fait passer de la confession amoureuse à sa dénégation par le truchement d'un travestissement identitaire et générique. Ce faisant, elle contribue à illustrer la plasticité des pratiques épistolaires dont fait preuve Hélisenne dans le recueil en adoptant diverses postures éthiques (en tant que consolatrice, conseillère, amoureuse impénitente ou défenderesse des droits des femmes) qui empruntent de diverses façons à la formalisation de la contentio orationis ${ }^{51}$. La narrativité résultant de la succession de ces postures fait du recueil moins une simple compilation qu'une forme de protoroman épistolaire ${ }^{52}$, soumis à l'esthétique de la varietas, chère aux humanistes, qui est d'ailleurs annoncée dans le préambule du recueil comme le prin-

49. Pour ce faire, elle en vient même à donner la parole à son mari qui lui fournit les arguments qu'elle retournera à son profit dans la troisième invective. À ce sujet, voir mon article «La fonction du dialogue épistolaire dans les Epistres invectives d'Hélisenne de Crenne», dans Benoît Melançon et Pierre Popovic (sous la dir. de), Les femmes de lettres. Écriture féminine ou spécificité générique?, Montréal, CULSEC, Département d'études françaises, Université de Montréal, 1994, p. 7-19.

50. À ce propos, voir notamment Jerry C. Nash, «Discours performatif d'une femme écrivain "exerceant œuvres viriles" : Les Epistres familieres et invectives d'Hélisenne de Crenne», dans Hélisenne de Crenne, ouvr. cité, p. 152-167.

51. Luc Vaillancourt, La lettre familière, ouvr. cité, p. 193.

52. Le recueil d'Hélisenne produit un effet narratif à partir des seules lettres, tandis qu'un autre protoroman épistolaire contemporain, Le jardin amoureulx. Contenant toutes les reigles d'amours (Paris, Alain Lotrian, vers 1537) de Christofle de Barrouso, inscrit les vingt lettres en prose qu'échangent l'amant et une dame dans un cadre narratif plus large qui emprunte le vers pour s'exprimer; au sujet de ce prosimètre, voir Yves Giraud, "L'étrange Jardin amoureux de maître Barrouso ", dans James Dauphiné et Béatrice Périgot (sous la dir. de), Conteurs et romanciers de la Renaissance. Mélanges offerts à Gabriel-André Pérouse, Paris, Honoré Champion, 1997, p. 209-224. À la suite de Bernard Bray, Yves Giraud semble considérer les dix-neuf épîtres amoureuses d'Étienne Pasquier (Recueil des Rymes et Proses, 1555) comme l'un des premiers romans épistolaires, méconnaissant l'antériorité de l'ouvrage d'Hélisenne ( «La dimension épistolaire dans quelques ensembles épistolaires du XVI e siècle», art. cité, p. 88). 
cipe moteur de l'écriture épistolaire d'Hélisenne (EFI, p. 55). Entre l'espace discursif familier des premières lettres et l'espace public de l'invective, obéissant tous deux à des codes clairs, la treizième lettre familière introduit le discours amoureux sur un mode ludique et indirect, au moyen d'une «imposture " constituant une façon paradoxale de décliner le moi amoureux, astreint à une rhétorique épistolaire qui est celle du simulacre. 